\title{
Overlapping Neural Correlates of Reading Emotionally Positive and Negative Adjectives
}

\author{
Traute Demirakca ${ }^{1,3}$, Cornelia Herbert ${ }^{1}$, Johanna Kissler ${ }^{2}$, Matthias Ruf ${ }^{3}$, Tim Wokrina ${ }^{3}$ and \\ Gabriele Ende ${ }^{*}, 3$ \\ ${ }^{1}$ Heidelberg Academy of Science, ${ }^{2}$ University of Konstanz, ${ }^{3}$ Central Institute of Mental Health, Department \\ Neuroimaging, J5, 68159 Mannheim, Germany
}

\begin{abstract}
Comparison of positive and negative naturally read adjectives to neutral adjectives yielded an overlapping higher BOLD response in the occipital and the orbitofrontal cortex (gyrus rectus). Superior medial frontal gyrus and posterior cingulate gyrus showed higher BOLD response to negative adjectives and inferior frontal gyrus to positive adjectives. The overlap of activated regions and lack of pronounced distinct regions supports the assumption that the processing of negative and positive words mainly takes place in overlapping brain regions.
\end{abstract}

\section{INTRODUCTION}

Emotional communication is a complex interaction of several features (e.g. prosody, content, face, gesture, body posture). Words acquire emotional content or meaning through association with other emotional stimuli such as visual images. This enables language to convey the pleasant or unpleasant nature of an object [1] and to elicit positive and negative emotions [2].

In contrast to pictorial emotional stimuli as faces and pictures processing of the emotional significance of words will require not only appraisal but also higher semantic processes. However, neuroimaging studies on emotional word processing report activation in brain regions similar to those investigating the processing of pictures and faces [3, 4].

The majority of studies concerning the neural correlates of processing of emotional verbal stimuli generally report the contrast of negative and positive emotional to neutral stimuli but the issue of overlap between the activated regions has not been discussed. Since only few studies have investigated the direct comparison of negative and positive stimuli the answer to the question concerning the overlap or distinctness of brain regions responsible for processing these stimuli is vague. A survey $[5,6]$ of the present studies found evidence for both, overlapping and distinct regions.

Considering the results of the studies that report a direct comparison of negative and positive lingual stimuli we expect distinct neural response to negative and positive words in several regions. Positive adjectives compared to negative adjectives are expected to lead to a higher BOLD response in the hippocampus [7] and the lingual gyrus [3, 7]. Negative words will result in stronger hemodynamic responses than positive words in the lateral prefrontal cortex, the superior temporal and the inferior parietal gyrus $[3,7]$.

\footnotetext{
*Address correspondence to this author at the Central Institute of Mental Health, Department Neuroimaging, J5, 68159 Mannheim, Germany;

Tel: +49-621-1703-2971; Fax: +49-621-1703-2005;

E-mail: gabi.ende@zi-mannheim.de
}

On the other hand the overlap of regions related in processing of negative and positive words has not been shown directly and can only be anticipated from the results of different studies. The following section will provide the regions with expected overlap for emotional processing of positive and negative stimuli.

Emotional words provoke in contrast to neutral words neural activity in frontal regions. The superior medial frontal gyrus is engaged in the generation and processing of emotional semantic information $[3,6,8-10]$. The inferior frontal gyrus is suggested to be employed in the evaluation of the emotional meaning of words comparable to the lateral frontal cortex, which is assumed to be responsible not only for working memory but also for retrieval of semantic information $[8,9,11]$. Emotional stimuli, irrespectively of their valence are processed in the bilateral orbitofrontal cortex [7, 8, 11-13]. The orbitofrontal gyrus has bidirectional connections with the amygdala and therefore is assumed to influence the attentional system [13]. Accounting the valence of the stimuli, all these frontal regions show enhanced activation in contrast to neutral words [1-3, 7, 12] and are suggested to process concomitant negative and positive stimuli. The majority of neuroimaging studies on emotional word processing failed to detect activation of the most prominent region involved in emotion processing, the amygdala. Few studies observed increased activation for negative and positive verbal material $[2,3,12]$ compared to neutral words. In summary most listed regions show activation during processing of emotional stimuli independent of the emotional valence. Only few regions seem to respond specifically to positive verbal stimuli as the putamen and nucleus accumbens which are suggested to be related to positive affect and reward [2].

The posterior cingulate as part of the cortico - limbic circuit has strong connections to the other regions related to emotional behavior and its activation is associated with the processing of emotional significance and memory related activity $[1,12]$. But activation differences of the anterior and posterior cingulate gyri are reported for both direct positive to negative contrast and emotional to neutral comparison $[1$, $7,12]$ and a preference hypothesis can not be conducted. 
Imaging studies investigating emotional language processing have used tasks that vary in the extent to which they focus the attention of the subject on the emotion. The tasks range from animacy/commonality judgment [3] to go/nogo [11] and lexical decision [7, 14], word generation $[1,9,10]$, valence evaluation [12] and paying attention to elicited feelings [2]. The influence of task effects on the activation pattern can not be estimated. Previous imaging studies found that cognitively demanding tasks can lead to a reduced amygdala BOLD response compared to passive viewing of faces or pictures [15]. Thus it can be assumed that passive or natural reading minimizes the effects of the task on neural response. The aim of the present study was to identify the overlap or diversity of brain regions involved in the processing of positive and negative adjectives with a natural reading task to minimize the task specific cognitive effects.

\section{METHODS}

\section{Stimuli}

$3 \times 34$ highly arousing pleasant and negative and low arousing neutral adjectives were used as visually presented stimuli. The adjectives with the highest valence and arousal ratings were drawn from a larger sample of 500 adjectives previously rated by 45 subjects for valence and emotional arousal on the Self Assessment Manikin Scale [16, 17]. Words from each category were comparable in word length and frequency in spoken language and positive and negative adjectives were balanced for emotional arousal. Stimuli were presented for $1 \mathrm{~s}$ in black letters on light gray background. During the jittered interstimulus interval ( 7.5 to $12 \mathrm{~s}$ ) the non-word , $x_{x x x}$ ' was presented as baseline condition. The subjects were instructed to read the presented words silently. Upon completion of the fMRI scans subjects were asked to write a list with all remembered words. The mean number of correctly retrieved adjectives was 11 with a range from 5 to 27.

\section{Participants}

22 healthy volunteers (mean age 40 years (STD 11), range 23-64 years, 3 males) participated in this study. All subjects were right-handed and native German speakers and had no history of psychiatric or neurological disease. All subjects provided written informed consent for the participation in the study. The study was approved by the Ethics Committee of the Clinical Faculty Mannheim of the University of Heidelberg, Germany.

\section{Imaging}

Functional MRI was performed on a $1.5 \mathrm{~T}$ Magnetom Vision Scanner (Siemens, Erlangen, Germany). Two runs with 135 and 185 scans were acquired using an echo planar imaging (EPI) sequence. Each volume consisted of 28 axial slices of $4 \mathrm{~mm}$ thickness (1 mm gap), TR $3 \mathrm{~s}$, TE $39 \mathrm{~ms}$, FOV $220 \times 220 \mathrm{~mm}^{2}, 64^{2}$ matrix and was angulated along the AC-PC plane. The first 3 volumes of each run were discarded to minimize T1 effects.

\section{Image Processing}

Data preprocessing and statistical analysis was performed with SPM2 (Wellcome Department of Cognitive Neuro$\operatorname{logy})$. All functional images were realigned to the first image, slice time correction was applied and the images were normalized to the standard template brain (MNI) provided by SPM. Finally the functional images were spatially smoothed with a Gaussian Filter (12 mm full width-half maximum) and the time series were high pass filtered (128 s). Motion parameters of each individual were included into the statistical model as covariates of no interest. Three different event types were included in the model for statistical analysis to model the valence of the adjectives (positive, negative and neutral). The regressors were convolved with a standard hemodynamic response function and its temporal derivative. Calculated fixed effect contrast images were entered into second level analysis. To asses the overlap of activated regions positive and negative items were compared to neutral ones and the overlap was confirmed by masking the contrasts inclusively with a threshold of $\mathrm{p}=0.01$. To discover regions that process distinct valences, negative and positive words were contrasted directly. Region of interest analyses was performed for the hypothesized regions. $\mathrm{T}$ values were considered statistically significant for a value of $\mathrm{p}<0.005$ (uncorrected for multiple comparisons) and a minimal cluster size of 10 contiguous voxels was applied. For the purpose of better visualization results are shown with a threshold of $\mathrm{p}=0.01$ (uncorrected).

\section{RESULTS}

The direct comparison between positive and negative adjectives resulted in stronger activation elicited by negative adjectives in the frontal superior medial gyrus (BA8, -3, 45, $54, \mathrm{k}=27, \mathrm{Z}=2.92, \mathrm{p}=0.002)$. This activation overlapped with the negative to neutral comparison for which the most notable focus of activation was found bilateral in the superior part of the medial frontal cortex (BA 8, 9), extending to SMA (BA6) (Fig. 1A, red spots). Additionally increased BOLD responses to negative adjectives were noted in the orbitofrontal region, more precisely in the left gyrus rectus (Fig. 1C, red spots). Further BOLD differences were observed in the middle occipital lobes, bilaterally (Fig. 1D, red spots), the left angular gyrus and the left posterior cingulate cortex (Fig. 1B, red spots). For local maxima see Table 1.

Reading positive adjectives compared to neutral ones resulted in an elevated BOLD response in similar but relatively smaller clusters (less than 30 voxel). As in the contrast comparing negative with neutral adjectives, these clusters were located in the middle occipital lobes, the orbitofrontal cortex (gyrus rectus) and the superior frontal cortex (see Fig. 1D, green spots). In contrast to the results observed for negative adjectives an increased BOLD response was also found for positive compared to neutral adjectives in the inferior frontal cortex (BA 47, Fig. 1C, green spots). Local maxima can be seen in Table $\mathbf{1}$. Conjunction or overlap of activated regions using the masking function confirmed that bilateral middle occipital lobe and left gyrus rectus were activated during processing of both negative and positive adjectives.

\section{DISCUSSION}

The study was designed to investigate hypothesized overlap in neural responses elicited by natural reading of negative and positive adjectives as compared to neutral adjectives and perform a direct comparison of the BOLD 


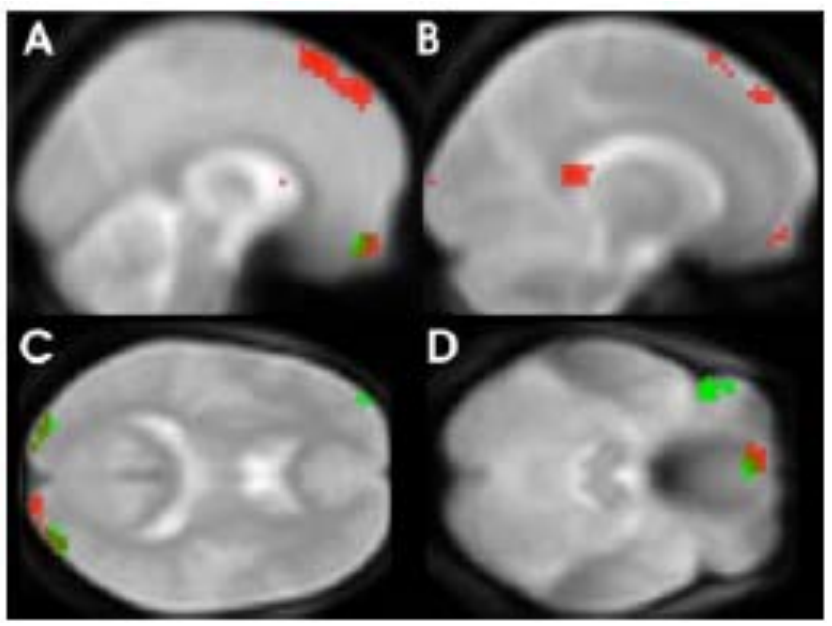

Fig. (1A-D): Areas with significantly greater BOLD response to negative adjectives (red spots) and positive (green spots) compared to neutral adjectives $(\mathrm{p}=<0.01$ for visualization purposes).

responses to negative and positive words. There was an essential overlap of activation in brain regions involved in the processing of positive and negative adjectives. The superior part of medial frontal gyrus was the only brain region showing significantly higher BOLD responses to negative than to positive words. Negative items caused a higher BOLD response in that region than both neutral and positive ones. Activation in the superior medial frontal cortex has been previously reported in several imaging studies investigating the processing of emotionally positive and negative words [7-9]. Two of these studies found an emotion-neutral difference during a semantic monitoring task [9] and a passive reading task [8]. In contrast Kuchinke et al. (2005) report stronger activation of this region by positive as opposed to negative and neutral adjectives during a lexical decision task [7].

Negative adjectives elicited stronger activation within the posterior cingulate cortex when compared to neutral adjectives. Posterior cingulate cortex activation by emotionally salient compared to neutral stimuli has been reported in imaging studies investigating healthy subjects [12] and enhanced activity of posterior cingulate structures has also been shown in patients with emotional disorders, rendering these posterior cingulated brain structures as candidates for a mood congruent bias [18] .

Two brain regions showed an essential overlap for both negative and positive adjectives as compared to neutral ones. The first cluster was located in the visual cortex (BA18, 19) in line with a previous imaging study reporting an elevated BOLD response in the occipital lobe (BA 18, 19) [8] during passive reading of emotional items. This kind of activation difference was also observed in studies investigating the processing of emotional faces (for an overview see [4]) suggesting that visual emotional stimuli are processed in a facilitated manner in the visual cortex. The second region with overlap for the emotional processing contrasts is the orbitofrontal cortex. Elevated BOLD responses in the orbitofrontal cortex (gyrus rectus) to emotional words irrespective of emotional valence have been found in several studies [7, 12] using written verbal emotional stimuli, but

Table 1. Significant Higher BOLD Response to Negative Compared to Neutral and Positive Compared Neutral Adjectives

\begin{tabular}{|c|c|c|c|c|c|c|c|c|c|}
\hline & $\begin{array}{l}\text { FEW } \\
\text { ROI }\end{array}$ & $\begin{array}{l}\text { FDR } \\
\text { ROI }\end{array}$ & $\begin{array}{l}\text { Cluster- } \\
\text { Size }\end{array}$ & $\mathbf{Z}$ & $\mathbf{x}$ & $\mathbf{y}$ & $\mathbf{z}$ & $\mathbf{p}$ & $\begin{array}{c}\text { Significant for } \\
\text { Masking Inclusively }\end{array}$ \\
\hline \multicolumn{10}{|l|}{ negative>neutral } \\
\hline left middle occipital lobe & & & 21 & 3.38 & -27 & -99 & 18 & $<0.001$ & yes \\
\hline right middle occipital lobe & & & 27 & 3.63 & 33 & -96 & 15 & $<0.001$ & yes \\
\hline left gyrus rectus & 0.625 & 0.179 & 35 & 3.12 & -9 & 54 & -21 & 0.001 & yes \\
\hline left posterior cingulate gyrus & & & 21 & 3.15 & -15 & -42 & 15 & 0.001 & no \\
\hline right middle occipital lobe & & & 11 & 2.86 & 36 & -93 & 9 & 0.002 & yes \\
\hline left gyrus rectus & & & 13 & 2.90 & -3 & 45 & -21 & 0.002 & yes \\
\hline left middle frontal gyrus & & & 16 & 3.07 & -36 & 60 & 3 & 0.001 & no \\
\hline left inferior frontal / orbitofrontal gyrus & 0.718 & 0.426 & 26 & 3.35 & -45 & 36 & -15 & 0.002 & no \\
\hline left superior frontal gyrus & 0.731 & 0.426 & 12 & 2.95 & -15 & 42 & 45 & 0.002 & yes \\
\hline
\end{tabular}

Region of interest (ROI) for overlap: orbifrontal cortex, gyrus rectus, superior medial frontal cortex, inferior frontal cortex, amygdala. 
has not been found in studies that used word generation or semantic monitoring $[1,9,10]$.

Activation of the amygdale by emotional stimuli is most frequently reported in studies using emotional faces and pictures [15] and in the emotion research literature the amygdala has been pinpointed to be a key structure involved in the facilitated processing of emotional salient stimuli. On the other hand, among the studies using verbal stimuli, only two studies report amygdala activation in response to verbal stimuli $[2,3]$. In the current study amygdala activation was absent not only in the direct comparison of emotional and neutral adjectives but also when considering the effects of interest. The amygdala facilitates perceptual vigilance for emotional stimuli. However, single word presentation may induce lower arousal than presentation of single emotional picture stimuli during passive viewing.

We did not find an activation of the anterior cingulate cortex. However, other regions of the limbic system (orbitofrontal cortex, posterior cingulate cortex) are activated by emotional words suggesting that emotional processing enhances brain activity in regions closely connected with the amygdale. Beauregard showed in a PET study that emotionally relevant material produces further processing in limbic brain structures of the orbito-frontal lobe [8].

Beside the missing amygdala activation our passive adjectives reading task leads to an activation pattern found similarly in other studies investigating emotional word reading and processing, respectively.

With the passive reading task, it is impossible to control what subjects associate with it. Viewing single words produces a cognitively complex mental state in which anticipation, emotional responses, visual perceptual analysis and activation of orthographic representation occurs [8]. None of these responses can be separately and/or individually determined.

\section{CONCLUSIONS}

The pattern of activated regions showed essential overlap with areas found in other studies and it can be suggested that silent reading of emotional adjectives induces emotional processing. Further studies have to evaluate the role of the task.

The overlap of activation of emotional compared to neutral adjectives and the negligible difference between positive and negative adjectives gives evidence that in healthy controls concordant brain regions are responsible for processing positive and negative items.

\section{ACKNOWLEDGEMENT}

This research was supported by a grant from the Heidelberg Academy of Science (Mind and Brain Program)

\section{REFERENCES}

[1] Cato MA, Crosson B, Gökcay D, et al. Processing words with emotional connotation: an fmri study of time course and laterality in rostral frontal and retrosplenial cortices. J Cogn Neurosci 2004; 16: 167-77.

[2] Hamann S, Mao H. Positive and negative emotional verbal stimuli elicit activity in the left amygdala. Neuroreport 2002; 13: 15-9.

[3] Kensinger EA, Schacter DL. Processing emotional pictures and words: effects of valence and arousal. Cogn Affect Behav Neurosci 2006; 6: 110-26.

[4] Vuilleumier P, Pourtois G. Distributed and interactive brain mechanisms during emotion face perception: evidence from functional neuroimaging. Neuropsychologia 2007; 45: 174-94.

[5] Ende G, Demirakca T, Tost H. The biochemistry of dysfunctional emotions: proton MR spectroscopic findings in major depressive disorder. Prog Brain Res 2006; 156: 481-501.

[6] Ethofer T, Pourtois G, Wildgruber D. Investigating audiovisual integration of emotional signals in the human brain. Prog Brain Res 2006; 156: 345-61.

[7] Kuchinke L, Jacobs AM, Grubich C, Vo ML, Conrad M, Herrmann M. Incidental effects of emotional valence in single word processing: an fMRI study. Neuroimage 2005; 28: 1022-32.

[8] Beauregard M, Chertkow H, Bub D, Murtha S, Dixon R, Evans A. The neural substrate for concrete, abstract, and emotional word lexica: a positron emission tomography study. J Cogn Neurosci 1997; 9: 441-31.

[9] Crosson B, Cato MA, Sadek JR, et al. Semantic monitoring of words with emotional connotation during fMRI: contribution of anterior left frontal cortex. J Int Neuropsychol Soc 2002; 8: 607-22.

[10] Crosson B, Radonovich K, Sadek JR, et al. Left-hemisphere processing of emotional connotation during word generation. Neuroreport 1999; 10: 2449-55.

[11] Elliott R, Rubinsztein JS, Sahakian BJ, Dolan RJ. Selective attention to emotional stimuli in a verbal go/no-go task: an fMRI study. Neuroreport 2000; 11: 1739-44.

[12] Maddock RJ, Garrett AS, Buonocore MH. Posterior cingulate cortex activation by emotional words: fMRI evidence from a valence decision task. Hum Brain Mapp 2003; 18: 30-41.

[13] Vuilleumier P. How brains beware: neural mechanisms of emotional attention. Trends Cogn Sci 2005; 9: 585-94.

[14] Nakic M, Smith BW, Busis S, Vythilingam M, Blair RJ. The impact of affect and frequency on lexical decision: the role of the amygdala and inferior frontal cortex. Neuroimage 2006; 31: 175261.

[15] Phan KL, Wager TD, Taylor SF, Liberzon I. Functional neuroimaging studies of human emotions. CNS Spectr 2004; 9: 258-66.

[16] Bradley MM, Lang PJ. Measuring emotion: the Self-Assessment manikin and the semantic differential. J Behav Ther Exp Psychiatry 1994; 25: 49-59.

[17] Herbert C, Kissler J, Junghöfer M, Peyk P, Rockstroh B. Processing of emotional adjectives: evidence from startle EMG and ERPs. Psychophysiology 2006; 43: 197-206.

[18] Drevets WC. Neuroimaging studies of mood disorders. Biol Psychiatry 2000; 48: 813-29. 